\title{
Fiber Changes Our Life
}

\section{Meifang Zhu ${ }^{1} \cdot$ Takeshi Kikutani $^{2} \cdot$ Tianxi Liu $^{1} \cdot$ Seeram Ramakrishna ${ }^{3} \cdot$ Guangming Tao ${ }^{4}$}

Received: 18 June 2019 / Accepted: 18 June 2019 / Published online: 9 August 2019

(c) Donghua University, Shanghai, China 2019

It is with great enthusiasm that we introduce the inaugural issue of Advanced Fiber Materials, a peer-reviewed, international and interdisciplinary research journal published by Springer Nature Publisher. Our aim is to provide a framework for research focused on fiber and Fiber-related expanses.

Fibers are synonymous with the history of mankind. Woven textiles from natural fibers such as cotton, hemp, linen, silk and wool have been used by the earlier humans in almost all world civilizations. Later on, chemical fibers, nano-fibers, functional fibers, and fiber-derived devices emerged.

Since the last century, silica as well as plastic optical fibers have played a significant role in "shrinking our world". With the development of science and technology, the scope of artificial and synthetic fibers has experienced a rapid transformation, leaving fibers deeply changing the world day by day. Essentially, the future of fibers should be more seamlessly integrated into our daily lives.

Fiber technology with universality and interdisciplinarity has been widely used in other research fields, including chemistry, condensed matter physics, engineering, materials, polymer science, renewable and green energy, and more. However, a specific academic publication which can

Meifang Zhu

zmf@dhu.edu.cn

Takeshi Kikutani

kikutani.t.aa@m.titech.ac.jp

Tianxi Liu

txliu@fudan.edu.cn

Seeram Ramakrishna

seeram@nus.edu.sg

Guangming Tao

tao@hust.edu.cn

1 State Key Laboratory for Modification of Chemical Fibers and Polymer Materials, College of Materials Science and Engineering, Donghua University, 2999 North RenminRoad, Songjiang District, Shanghai 201620, China provide a platform for these fiber and fiber-related cuttingedge research progress is still lacking. Therefore, the launching of "Advanced Fiber Materials" is timely and critically important in scientific, social and economic progress. We aim to provide authors with venues to exchange their ideas, describe and analyze their fiber research as well as applications from their perspectives.

The journal scope as mentioned in the instructions to authors is as follows:

"Advanced Fiber Materials is a peer-reviewed, international and interdisciplinary research journal which publishes original and review articles on fiber and fiber-related device as well as their applications, including carbon nanofibers, natural fibers, smart fibers, high-performance fibers, multifunctional and composite fibers, environment-friendly fibers as well as other fiber-related and fiber-shape materials and devices (fiber-based artificial skins for robots and wearable intelligent devices). Novel approaches to synthesis, characterization, and/or applications of new and existing fibers that enable fiber technology develop rapidly, are also within the journal scope."

Advanced Fiber Materials is launched in 2019 by State Key Laboratory for Modification of Chemical Fibers and Polymer Materials, Donghua University (China). Accepted

2 Department of Materials Science and Engineering, Tokyo Institute of Technology, 2-12-1, Ookayama, Meguro-ku, Tokyo 152-8550, Japan

3 Centre for Nanofibers and Nanotechnology, National University of Singapore, 3 Science Drive 3, Singapore 117543, Singapore

4 Wuhan National Laboratory for Optoelectronics and School of Optical and Electronic Information, Huazhong University of Science and Technology, Luoyu Road 1037, Wuhan 430074, China 


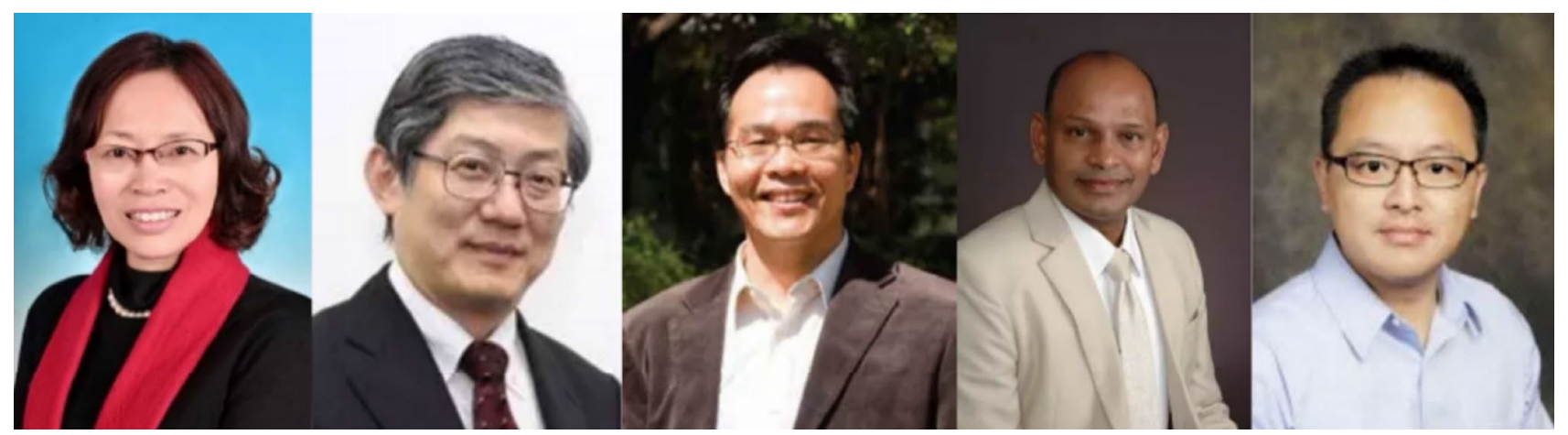

Advanced Fiber Materials editors (left to right): Editor-in-chief: Prof. Meifang Zhu, Donghua University, China. Associate editor: Prof. Takeshi Kikutani, Tokyo Institute of Technology, Japan. Associate editor: Prof. Tianxi Liu, Donghua University, China. Associate editor: Prof. Seeram Ramakrishna, National University of Singapore, Singapore. Associate editor: Prof. Guangming Tao, Huazhong University of Science and Technology, China

Fig. 1 Editorial board members

article types include Research Article, Review, Letter, News, Perspective and Highlight.

I, Prof. Meifang Zhu, am delightful to have been selected as the Editor-in-chief and pleased to be working with Prof. Takeshi Kikutani, Prof. Tianxi Li, Prof. Seeram Ramakrishna and Prof. Guangming Tao to serve this Journal (Fig. 1).

As editors, we look for the ground-breaking and innovative findings that involve fibers and encourages the exchange of ideas among chemists, physicists, material scientists, energy/environmental/biomedical researchers, engineers and other researchers who are active at the frontiers of all fiberrelated fields. We hope that by having publications on all aspects of this important area of research in the one journal, discussions will be initiated between researchers who might not otherwise meet and that this may lead on to new research strategies and insights.

Now, just enjoy the first issue and do not forget that we are sincerely looking forward your submission with your remarkable research on fibers. We believe that Advanced Fiber Materials will serve as the bridge for fiber scientists, engineers and entrepreneurs all over the world.
Prof. Meifang Zhu comes from Donghua University (China), has over 30 years of research experience in organic and inorganic hybrid materials, nanocomposite hydrogel materials, fiber forming processing and theory, basic research and application development of biomass fiber and biomedical materials.

Prof. Takeshi Kikutani comes from Tokyo Institute of Technology (Japan) and his fields of specialization involve polymer processing (melt spinning), structure and property of fibers and polymers as well as fiber reinforced composites.

Prof. Tianxi Liu comes from Donghua University (China) and is committed to polymer nano-composite materials, new energy materials and devices, organic-inorganic hybrid materials, electrostatic spinning nano-fiber composite materials.

Prof. Seeram Ramakrishna comes from National University of Singapore (Singapore) and his research interests include polymer composites, carbon nanofibers, electrospinning, nanofibers for energy, environment, healthcare and wearables applications.

Prof. Guangming Tao comes from Huazhong University of Science and Technology (China) and is mainly engaged in functional fibers, smart fabric, man-machine interactions, specialty optical fibers and in-fiber nano-fabrication. 